\title{
The Application of Plasma Processes during the Production of Pigments
}

\author{
Alexander Vyboishchik ${ }^{1, *}$, and Mikhail Popov ${ }^{1}$ \\ ${ }^{1}$ South Urals State University, Russia, 454080, Chelyabinsk, Lenin Ave. 76
}

\begin{abstract}
The article describes the application of plasma in the production of industrial pigments. Plasmatrons, or cooled electrodes, have found practical use in chemical, petrochemical, metallurgical, and other industries. Plasma-chemical methods with the use of plasmatrons have found application in the yielding of high-quality pigments, e.g. titanium dioxide. The disadvantage of the application of plasmatrons is the dilution of the outgoing chlorine with inert gases, which reduces the service life of plasmatrons. The disadvantage can be eliminated by substituting high frequency induction plasma with arc plasma.
\end{abstract}

\section{Introduction}

Plasma is an ionized gas containing charged particles, i.e. unbound electrons and ions. Gaseous plasma differs from any system of freely moving charged particles in quasineutrality: the positive charge of ions and the negative charge of electrons are mutually neutral on the average.

There exist various areas of application of plasma processes: in chemical industry for producing polymers $[1,2]$, removing corrosion layers [3], residuals [4], powders [5, 6], deposition of films and membranes [7, 8, 9], separation of isotopes [10], obtaining metals and metal oxides $[11,12,13,14,15,16,17]$, biotechnology [18], microbiology, medical technology [19], geophysics [20], textile industry [21]. One of the most challenging trends of civil engineering is the production of pigments used as paints as coatings.

\section{General characterization of plasma processes}

A system of charged particles is named plasma only if the size of such system is large enough compared to Debye length, and the discernible separation of charges in plasma is possible only at short wavelengths or for short periods of time, so that its lifetime is longer than the period of plasma oscillations [22, 23].

As is known, any substance at sufficiently high temperatures, as well as high-density gases transform into a plasma state. The higher the ionization energy, the higher temperatures are required for ionization. The lowest ionization energy is required for alkali metals. Chemical processes in low-temperature plasma are possible in the presence of non-

\footnotetext{
* Corresponding author: alex_vyb@list.ru
} 
dissociated molecular ions. Molecules in gas-discharge plasma enter into various chemical reactions. In a state of dense equilibrium plasma, reactions proceed according to a purely thermal mechanism, whereas the electric discharge acts as a simple heater. The obtaining of ozone in an electric discharge is an example of such a process.

To apply plasma processes in practice, various plasma-chemical devices are used [24, 25]. An ordinary plasma-chemical device works as follows: the source substance in a liquid, sintered or slurry state is fed into a stream of low-temperature plasma ( $3000 \mathrm{~K}$ or higher). At this temperature in the reactor, the substance decomposes into molecules, atoms, and ions. Due to the controlled composition of orifice gases (hydrogen, nitrogen, oxygen, water vapour), there are formed oxides, hydrogen-halogen compounds, neutral molecules and atoms in accordance with the required thermodynamic processes. Such processes are strictly regulated in the plasma-chemical device by pressure, temperature and gas composition.

The high temperature of the gas, as well as the systems of gas quenching, neutralization, regeneration, filtration systems, and, finally, the process terminability at any time make the under-pressure processes environmentally friendly albeit power-consuming.

The most important trends of the research of plasma processes aim at solving the following promising energy problems: thermonuclear controlled reactions and direct thermal-to-electrical energy conversion. The most important is the interaction process between various reagents and plasma jets. Thus, plasma torches have found practical use in electric welding; high-frequency induction-plasma torch burners have found practical use in crystal growing process of heat-resistant substances in refractory and metallurgical branches of industry. Complex undecomposable mineral molecules transform into a plasma state and, therefore, become easily ionizable (e.g., rhodonite $\mathrm{MnO} \cdot \mathrm{SiO}_{2}$ ).

Ionization process in chemical, petrochemical, metallurgical, and other industries use powerful arc discharges with cooled electrodes, or plasmatrons. Plasma technologies allow designing both mobile and stationary industrial devices for both processing of natural raw materials and recycling of various wastes. For example, the complete electrophysical process for the recycling of machine-building waste (iron chips, filings, cast iron) with the yield of iron-containing pigments of red iron oxide has been developed. This type of device transforms waste into raw materials for ceramic products, as well as adsorbents, heatinsulating materials, etc. [26].

Plasma processes are also applied in solder-bath waste dehydration. However, such processes are more dangerous, although they reduce the mass of the equipment and improve the energy performance of the process.

Recently, there have been both invented industrial tests and implemented recycling processes for raw materials and industrial waste with the use of plasmatrons. For example, tests performed on an ore-thermal furnace with the use of 150 and $200 \mathrm{~kW}$ plasma arc plasmatrons for reductive slag depletion have showed positive results. The process of plasma recovery of slag surpasses its well-known analogues in technical and economic parameters. The use of a $1 \mathrm{MW}$ plasmatron blasting unit allows developing, alongside with liquid furnace autogenous smelting, the high-performance process of depletion of slag of various compositions and properties. The use of plasmatrons for the oxidation of titanium tetrachloride with oxygen in order to obtain titanium dioxide is also a promising trend.

\section{Application of plasma processes for the yield of pigments}

To obtain high quality pigments, especially titanium dioxide, there are applied plasmachemical methods, the essence of which is to heat an inert or oxygen-containing gas in an arc or induction plasmatron to a temperature in the range from 2000 to $12000 \mathrm{~K}$, followed by the feeding of oxygen and titanium tetrachloride into the obtained plasma jet. There are 
known various applications of such plasma-chemical method; for example, an inert gas is heated in two arc plasmatrons, and then oxygen and titanium tetrachloride are fed into each plasma stream separately, and, subsequently, sent to a common reactor for mixing and oxidation. One reagent can be superheated to a high temperature, while the second, e.g. liquid titanium tetrachloride, is fed into the reactor without heating. The method allows maintaining very high temperatures in the reaction zone; therefore, the formation of nuclei of a new rutile phase occurs without the feeding of special reagents. The staying time of the reactants in the reaction zone does not exceed $1 \mathrm{sec}$ (actually within the range from 0.02 to $0.1 \mathrm{sec}$ ). In the case of using an arc generator, its electrodes, viz. cathodes, are produced from specific materials (aluminium, titanium, zirconium carbides) so that electrodes can serve as modifying additives due to their erosion and subsequent interaction with oxygen. The schematic diagram of a simple plasmatron is given in Fig. 1.

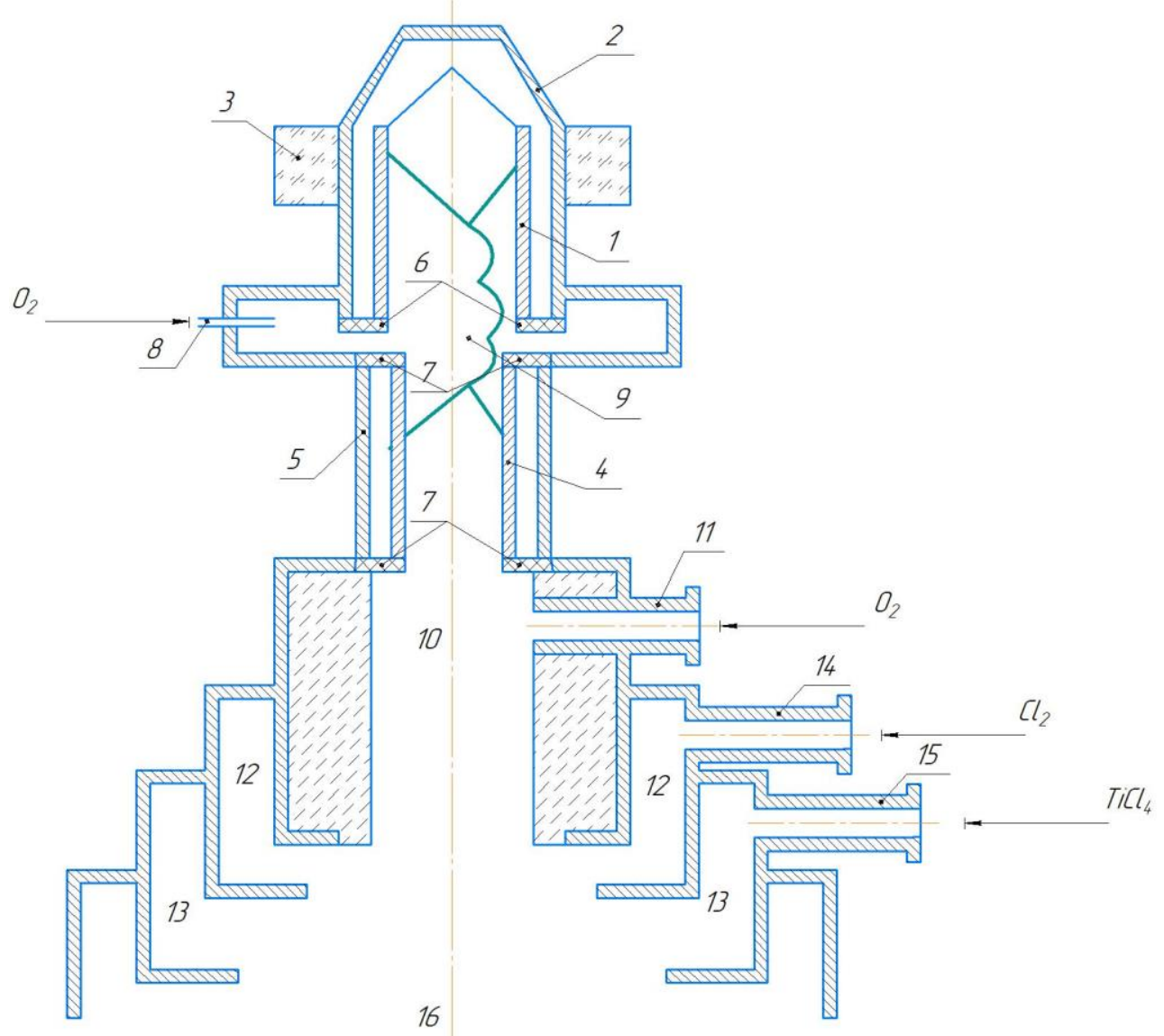

Fig. 1. Plasmatron: 1 - upper electrode, 2,5 - water-cooled jacket, 3 - voltaic arc stabilizing solenoid, 4 - lower electrode, 6,7 -insulators, 8 - hole, 9 - whirling chamber, 10 - mixing chamber, 11,14,15wave inlets, 12,13 - insulating chambers, 14 - wave outlet, 16 - reaction chamber.

The upper electrode 1 is provided with the water-cooled jacket 2, the upper part of which is embedded into the solenoid 3 designed to stabilize the voltaic arc, with the voltage ranging from 20 to $30 \mathrm{~V}$, and the amperage of $400 \mathrm{~A}$. The magnetic field created by the solenoid causes the shift and subsequent stabilization of the arc, which, in its turn, decreases the wear of the electrode 1 . The lower electrode 4 is also provided with the watercooled jacket 5 , and is located coaxially with the electrode 1 , but has a smaller diameter. 
The electrodes are based on the insulators 6 and 7. An oxygen-containing gas is fed through the hole 8 and the whirling chamber 9 located between the electrodes 1 and 4 . A voltaic arc arises between the electrodes 1 and 4, where oxygen is heated and partially ionized. In the chamber 10, located below the electrode 4, a hot oxygen stream is mixed with a perpendicularly directed secondary stream of oxygen fed through the wave inlet 11 , with the temperature of the mixture in the range from 1900 to $2500{ }^{\circ} \mathrm{C}$. The chamber 10 is provided with the concentrically embedded chambers 12 and 13. Cold chlorine is fed through the wave inlet 14 into the chamber 12 thus creating an insulating layer between the flow of oxygen and titanium tetrachloride previously fed into the reactor through the wave inlet 15 into the chamber 13. The chlorine layer prevents interaction between the reactants before they enter the reaction chamber 16. Various additives can be introduced together with the reactants.

The main disadvantages of this method are, firstly, the dilution of the outgoing chlorine gas with an inert carrier (argon, nitrogen, etc.) resulting in a sharp decrease in the service life of the arc plasmatron, and, secondly, an increase in the oxygen content in the orifice gas resulting an increase in the maintenance cost of the plasmatron. The use of high-frequency induction plasma in comparison with arc plasma has several advantages, since, besides inert gases (nitrogen, air, etc.), non-inert gases like oxygen, hydrogen, and even aggressive halogens can be subject to high-temperature heating also. The on-stream period of the highfrequency installation and the absence of wearing parts allows obtaining a plasma jet without pollution and, consequently, with no quality loss of the yielded products.

\section{Conclusions}

Plasma processes have found practical application in various branches of industry, viz. chemical, petrochemical, metallurgical, etc. On of practical implementation of plasma processes is recycling of raw materials and industrial wastes.

The use of plasmatrons allows yielding high quality pigments, e.g. titanium dioxide.

The disadvantage of using plasmatrons is the dilution of the outgoing chlorine gas with inert gases thus reducing both the service life and the maintenance cost of plasmatrons.

The above-mentioned disadvantage can be eliminated by using high frequency induction plasma instead of arc plasma.

\section{References}

1. Yuichi Setsuhara, Ken Cho, Kosuke Takenaka, Masaharu Shiratani, Makoto Sekine, Masaru Hori, Advanced research and development for plasma processing of polymers with combinatorial plasma-process analyzer, Thin Solid Films, v. 518(22), pp. 63206324 (2010)

2. L.I. Kravets, Sergey N. Dmitriev, Gheorghe Dinescu, V. Sleptsov, V. Elinson, Modification of Poly(Ethylene Terephthalate) Track Membrane Properties by Plasma Chemical Method, Materials Science Forum, v. 636-637, pp. 805-811 (2010)

3. Zhenwei Wu, Runfei Liu, Xiaodong Du, Removing of sulfide corrosion layers by an arc plasma process, Materials Testing, v. 62(8), pp. 809-813 (2020)

4. G.G. Garifzyanova, G.G. Garifzyanov, Pyrolysis of vacuum resid by the plasma chemical method, Chemistry and Technology of Fuels and Oils, v. 42(3), pp. 172-175 (2006)

5. D. Neuschütz, Plasma processing of dusts and residues, Pure and Applied Chemistry, v. 68(5), pp. 1159-1165 (1996) 
6. Ilya Zhukov, Sergey Vorozhtsov, Vladimir Promakhov, Ivan Bondarchuk, A. Zhukov, A. Vorozhtsov, Plasma-chemical method for producing metal oxide powders and their application, Journal of Physics Conference Series, v. 652(1), pp. 012027 (2015)

7. R.G. Sharafutdinov, Petr A. Skovorodko, Victor Shchukin, V.O. Konstantinov, Silicon Film Deposition Using a Gas-Jet Plasma-Chemical Method: Experiment and GasDynamic Simulation, Journal of Applied Mechanics and Technical Physics, v. 59(5), pp. 786-793 (2018)

8. Victor Shchukin, R.G. Sharafutdinov, V.O. Konstantinov, Deposition of Silicon Films Doped with Boron and Phosphorus by the Gas-Jet Plasma-Chemical Method, Semiconductors, v. 53(1), pp. 127-131 (2019)

9. Victor Shchukin, V.O. Konstantinov, R.G. Sharafutdinov, Deposition of Amorphous and Microcrystalline Films of Silicon by the Gas-Jet Plasma-Chemical Method, Semiconductors, v. 53(12), pp. 1712-1716 (2019)

10. Il Gyo KOO, Woong Moo LEE, Hydrogen Isotope Separation by Plasma-Chemical Method, Journal of Nuclear Science and Technology, v. 42(8), pp.717-723 (2005)

11. Jian-Zhang Chen, Shih-Hang Chang, Plasmas Processes Applied on Metals and Alloys, Metals - Open Access Metallurgy Journal, v. 10(3), pp. 349 (2020)

12. G.G. Lepezin, A.S. An'shakov, V.A. Faleev, E.G. Avvakumov, O.B. Vinokurova, Plasma-chemical method for producing silumin and aluminum from sillimanite group minerals, Doklady Chemistry, v. 456(2), pp. 110-113 (2014)

13. Yu.M. Grishin, N.P. Kozlov, Andrey Skryabin, Efficiency of the plasma-chemical method of preparation of silicon from quartz in an argon-hydrogen flow, High Temperature, v. 54(5), pp. 619-626 (2016)

14. A. Kuzjukevics, S. Linderoth, J. Grabis, Characterization of Yttria-Doped Zirconia Powders Produced by Plasma-Chemical Method, Solid State Ionics, v. 92(3-4), pp. 253-260 (1996)

15. V.A. Nelyub, Plasma Processing of Fibrous Carbon Materials prior to Their Metallization, Polymer Science Series D, v. 13(4), pp. 429-434 (2020)

16. Jagadish Parida, Subash Chandra Mishra, Suresh Chandra Pattnaik, Wear Behavior of Plasma Processed LM6 Alloy, Materials Science Forum, v. 978, pp. 140-144 (2020)

17. N.V. Dedov, S.B. Tochilin, Yu.N. Tumanov, A.N. Zhiganov, Development of a Technology for Dissolving a Homogeneous Mixture of Uranium and Plutonium Oxides Obtained by the Plasma-Chemical Method, Inorganic Materials: Applied Research, v. 11(3), pp. $572-578$ (2020)

18. Bernd Kramer, D. Hasse, S. Guist, Thomas Schmitt-John, Peter Muranyi, Inactivation of bacterial endospores on surfaces by plasma processed air, Journal of Applied Microbiology, v. 128(4) (2019)

19. Klaus-Dieter Weltmann, Martin Polak, Kai Masur, Th. von Woedtke, Jörn Winter, Stephan Reuter, Plasma Processes and Plasma Sources in Medicine, Contributions to Plasma Physics, v. 52(7), pp. 644-654 (2012)

20. Sergey I. Popel, Dusty Plasma Processes in Geophysics, Plasma and Fusion Research, v. 4, pp. 31-031 (2009)

21. Sebastien Guimond, B. Hanselmann, Martin Amberg, Dirk Hegemann, Plasma processing of textiles: Perspectives, Technische Textilien, v. 53(4), pp. E130-E131 (2010)

22. L.L. Alves, Y. Ségui, Special issue on Plasma Processes, The European Physical Journal Applied Physics, v. 56(02) (2011) 
23. Albert Farrugia, Chris V. Prowse, Valerie Scott Hornsey, Conditions for plasma processing, Transfusion, v. 51(8), pp. 1875-6 (2011)

24. Klaus Landes, Plasma Generators for thermal plasma processes, International Journal of Materials Research (formerly Zeitschrift fuer Metallkunde), v. 102(8), pp. 959-963 (2011)

25. S.I. Ivanov, A.A. Petrenko, I.A. Dmitrieva, O.V. Sakharova, Equipment for plasma processing in C0 2, Journal of Physics Conference Series, v. 1399044103 (2019)

26. S.A. Shevchenko, L.P. Levlyuk, S.M. Pavlov, Application of ultrafine powders produced by plasma-chemical methods, Powder Metallurgy and Metal Ceramics, v. 23(6), pp. 425-430 (1984) 